\title{
Performance enhancement of dye-sensitized solar cells by incorporating graphene sheets of various sizes
}

\author{
Soumitra Satapathi ${ }^{a}$, Hardeep Singh Gill ${ }^{a}$, Sriya $\operatorname{Das}^{b}$, Lian $\mathrm{Li}^{c}$, Lynne Samuelson ${ }^{c}$, Micah J.Green ${ }^{b}$, and Jayant \\ $\operatorname{Kumar}^{a, *}$ \\ ${ }^{a}$ Center for Advanced Materials, University of Massachusetts Lowell, Lowell, Massachusetts,01854, USA \\ ${ }^{b}$ Department of Chemical Engineering, Texas Tech University, Lubbock, Texas, 79409, USA \\ ${ }^{c}$ U.S. Army Natick Soldier Research, Development \& Engineering Center, Natick, Massachusetts 01760, USA \\ *E-mail: Jayant_kumar@uml.edu
}

\begin{abstract}
Dye-sensitized solar cells (DSSCs) were fabricated using photoanodes made from graphene- $\mathrm{TiO}_{2}$ nanocomposites. The dependence of the size of graphene sheets on the cell performance was investigated. The experimental results indicated that cells loaded with the smaller graphene sheets yielded larger enhancement. The smaller graphene sheets improved the dye adsorption, leading to higher conversion efficiency. The DSSC incorporated with graphene sheets of $184 \mathrm{~nm}$ exhibited the largest enhancement in efficiency $(\sim 49 \%)$ as compared to the cell without graphene.
\end{abstract}

Key Words: Graphene, Dye sensitized solar cells, Dye-Loading, Porosity.

\section{Introduction}

Dye-sensitized solar cells (DSSCs), developed by Gratzel and co-workers, provide a promising alternative clean and renewable energy source [1]. DSSCs employing semiconducting titanium dioxide $\left(\mathrm{TiO}_{2}\right)$ and organometallic dyes can efficiently convert sun light into electricity. Power conversion efficiency upto $12 \%$ has been reported [2]. Numerous approaches have been taken so far to enhance the conversion efficiency, including design and synthesis of new sensitizers for better light-harvesting, fabrication of two of more DSSCs in tandem form for broader light absorption, development of novel electrolytes with more suitable redox potential for efficient dye regeneration and charge transport etc.[3-5] Other approaches, such as utilization of periodic nanostructures for effective light-trapping and metal nanoparticles for better light-absorption using surface plasmon resonance, have been adapted to improve the photovoltaic performance [6-8]. However, there still exist many possibilities for further improvements of the efficiency to meet the requirement for practical applications. Employing nanostructured carbon materials, such as graphene, would be an attractive alternative. Single layer graphene is highly transparent (only one atom thick) and absorbs only $2.3 \%$ of the incident light. In addition, graphene exhibits extremely high electron mobility $\left(15,000 \mathrm{~cm}^{2} /(\mathrm{V} \cdot \mathrm{s})\right)$ at room temperature [9]. Furthermore, graphene can be easily derived from graphite, which is naturally abundant and inexpensive. It is well-known that graphene possesses very high specific surface area $\left(2,630 \mathrm{~m}^{2} / \mathrm{g}\right)$ [10]. Its unique electronic and optical properties along with its high surface area and ease of synthesis render graphene as a suitable candidate for photovoltaic performance enhancement in DSSCs.

Graphene has recently been incorporated into the photoanodes in DSSCs [11-16]. It has been shown that the incorporation of graphene in the photoanodes leads to enhancement in light-harvesting, faster electron transport and reduces charge- recombination. Enhancement on the photovoltaic performance was achieved. It was reported that the DSSCs with a graphene loading of $0.83-1 \mathrm{wt} \%$ yielded the highest power conversion efficiency [13-14]. However, to the best of our knowledge, there is no report on the dependence of graphene sheet size on the device performance in the literature so far. Here, we report the size effect of graphene on the performance enhancement of DSSCs. As the graphene size decreases, both the short-circuit current and the efficiency increase. Dye desorption studies indicated improvement of dye loading in the photoanodes incorporated with smaller graphene sheets.

\section{Experimental}

The photoanode in a typical DSSC, is composed of 
Table I. Sizes and monodispersities of the graphen e sheets used in the DSSCs with or without sonic ation(double column)

porous semiconducting $\mathrm{TiO}_{2}$ nanoparticles (NPs) loaded with the sensitizers. The nanoporous photoanode also provides a percolation network for the passage of photogenerated electrons to the transparent conducting oxide substrate. In this study, we have modified the photoanode by heterogeneous mixing of $\mathrm{TiO}_{2} \mathrm{NPs}$ with graphene of various sizes. For better control over the graphene size, we have prepared the graphene dispersions using two different approaches, the modified Hummers method [17] and exfoliation of pristine graphene in the presence of stabilizers [18]. In the first approach, graphene was synthesized by the chemical reduction of graphene oxide with hydrazine monohydrate. Graphene oxide was first made by the modified Hummers method through acid oxidation of graphene nanoplatelets. Subsequently, graphene oxide dispersion was reduced to form reduced graphene oxide by hydrazine monohydrate at $80{ }^{\circ} \mathrm{C}$ for 2 hours, centrifuged to form homogeneous stable graphene dispersion and dried via evaporation of water.

In the second approach, expanded graphite was tip sonicated for 1 hour in surfactant solution (sodium dodecyl benzene sulfonate). The sonicated sample was then centrifuged for 4 hours at $5000 \mathrm{rpm}$ to remove large aggregates of graphite, and the supernatant was retained. The characterization of graphene dispersion was reported earlier [18]. Dynamic light scattering (DLS) was used to examine the size distribution of the graphene in the dispersions. A compact thin $\mathrm{TiO}_{2}$ layer (about $80 \mathrm{~nm}$ ) was spin-cast onto a fluorine-doped tin oxide (FTO) coated glass substrate by a sol-gel process [19]. The synthesized graphene was then used to fabricate $\mathrm{TiO}_{2}$-graphene composite photoanodes. Degussa P25 $\mathrm{TiO}_{2} \mathrm{NPs}$ suspension in acetic acid water was prepared. The zeta potentials of the graphene dispersion and the $\mathrm{TiO}_{2} \mathrm{NPs}$ suspension were measured. To investigate the size effect of the graphene sheets on the photovoltaic performance, the graphene prepared by the Hummers method were probe sonicated for different time duration to obtain smaller graphene sheets. The graphene produced by the second method with an average size of $184 \mathrm{~nm}$ was used without sonication. The $\mathrm{TiO}_{2}$ suspension was then mixed with $1 \mathrm{wt} \%$ of the graphene sheets. Nanoporous working electrodes were prepared by spin coating the mixture on the top of the thin $\mathrm{TiO}_{2}$ covered FTO substrates. The surface characteristics of bare photoanode and graphene loaded $\mathrm{TiO}_{2}$ photonaode were investigated by scanning electron microscopy (SEM).

The composite photoanodes ( $4 \mu \mathrm{m}$ thick) with different graphene sizes were used to fabricate the DSSCs. The spin-cast nanocomposite photoanodes were sintered at $450{ }^{\circ} \mathrm{C}$ for 4 hours and then dipped in $0.3 \mathrm{mM}$ Z907 dye ethanol solution overnight. The chemical structure of the dye is shown in Fig. 1. The

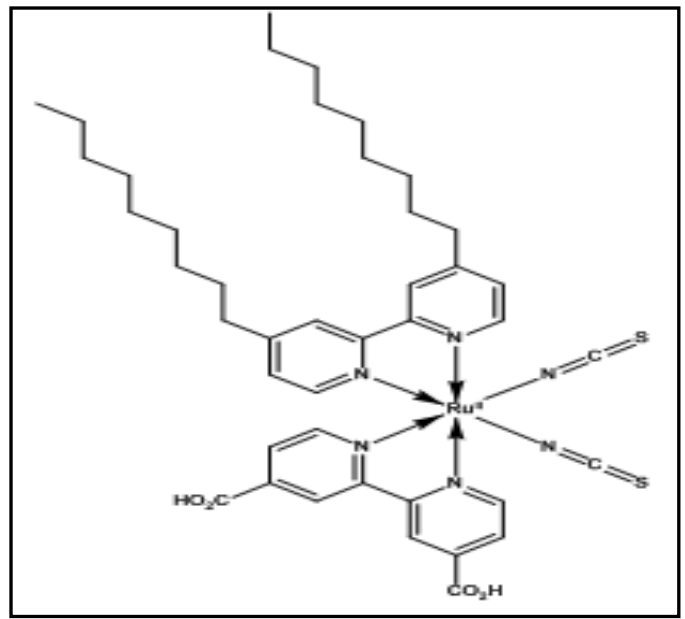

Figure 1. Chemical structure of Z907 dye.

platinum coated FTO substrates were used as counter electrodes. The DSSCs without any graphene loading were also fabricated as references (standard cells). The photovoltaic performance of the solar cells was evaluated under simulated AM 1.5 solar irradiation $\left(100 \mathrm{~mW} / \mathrm{cm}^{2}\right)$ in conjunction with a source meter.

\section{Results and Discussion}

Fig. 2(a) shows the cross-sectional SEM image of a $\mathrm{TiO}_{2}$ photoanode where a large amount of nanoparticles are densely packed. $\mathrm{The} \mathrm{TiO}_{2}$ film is 
highly porous. Fig.2 (b) shows the cross-sectional SEM image of the graphene- $\mathrm{TiO}_{2}$ composite film with $1 \mathrm{wt} \%$ graphene $(184 \mathrm{~nm})$ loading. The image revealed that the graphene sheets were well-dispersed within the the $\mathrm{TiO}_{2}$-NPs. Increase in porosity of the grapheneloaded nanocomposites as compared to that of the $\mathrm{TiO}_{2}$ films is observed. This led to improvement in the dye loading and to enhancement of the photocurrent in the DSSCs. The zeta potential analysis showed that the graphene surfaces were negatively charged $(-42 \mathrm{mV})$ whereas the $\mathrm{TiO}_{2}$ nanoparticles were positively charged (around $30 \mathrm{mV}$ ). When these two oppositely charged nanomaterials were mixed together, the electrostatic interaction would enable them to adhere on each other. Graphene sheets of different sizes were obtained by probe sonication of the Hummers method synthesized graphene at various time durations. Longer sonication yielded smaller graphene sheets. After 60 min of probe sonication, the size of the graphene decreased to $292 \mathrm{~nm}$. Further sonication for a considerable amount of time did not reduce the size of graphene significantly. The graphene with an average size of $184 \mathrm{~nm}$ prepared by the second method was utilized without sonication. Table I summarizes the sizes and monodispersities of the graphene sheets used in the DSSCs with or without sonication as determined by DLS.

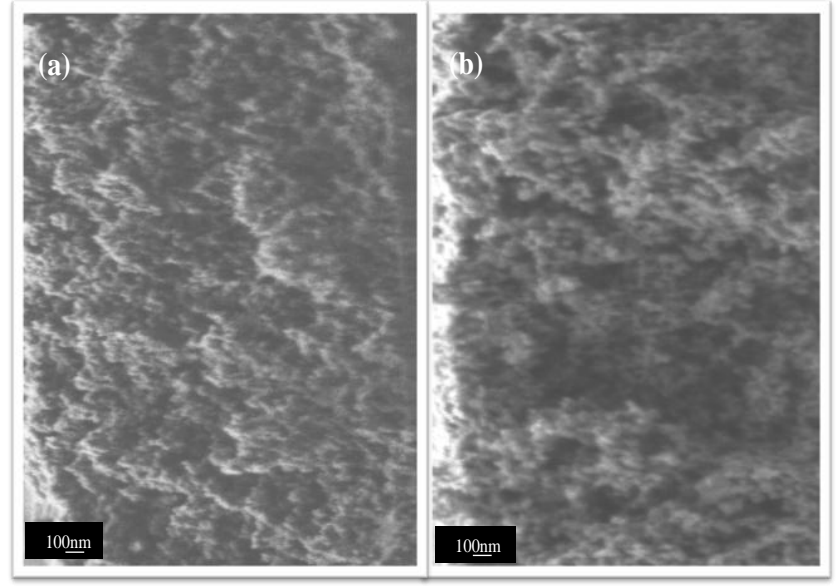

Figure 2. SEM images of (a) a $\mathrm{TiO}_{2}$ photoanode and (b) $\mathrm{a} \mathrm{TiO}_{2}$-graphene composite.

Fig. 3 shows the current-voltage (I-V) characteristics of the DSSCs. The standard $\mathrm{TiO}_{2}$ cell without any graphene loading yielded a short-circuit current density $\left(\mathrm{J}_{\mathrm{sc}}\right)$ of $9.76 \mathrm{~mA} / \mathrm{cm}^{2}$, an open circuit voltage $\left(\mathrm{V}_{\mathrm{oc}}\right)$ of $0.68 \mathrm{~V}$, a fill-factor (FF) of $66 \%$ with a power conversion efficiency $(\eta)$ of $4.43 \%$. Upon loading of the 1.2 micron size graphene sheets, $22 \%$ increase in $\mathrm{J}_{\mathrm{sc}}$ and $15 \%$ gain in $\eta$ were attained. $32 \%$ and $41 \%$ increase in $J_{\mathrm{sc}}$ were obtained using $444 \mathrm{~nm}$ and $292 \mathrm{~nm}$ size graphene, respectively. The highest photovoltaic performance enhancement was achieved by employing the graphene with an average size of $184 \mathrm{~nm}$. A $\mathbf{J}_{\mathrm{sc}}$ of

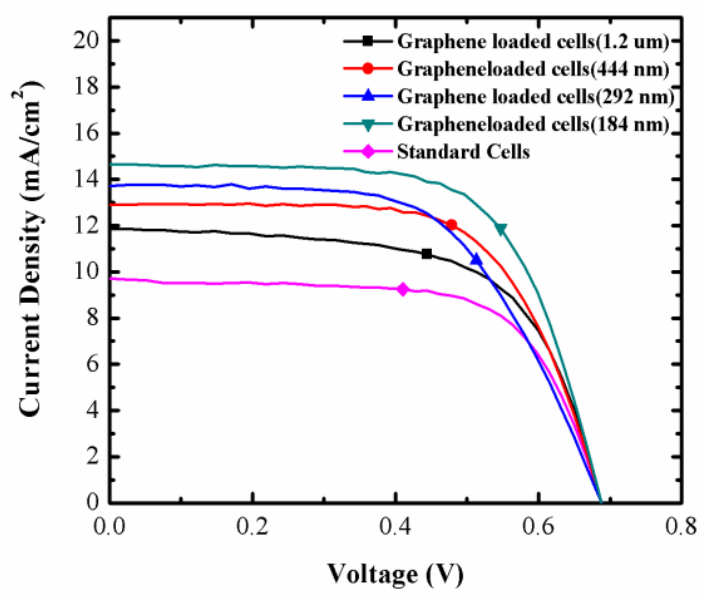

Figure 3. I-V curves of the DSSCs made with graphene of different sizes.

$14.66 \mathrm{~mA} / \mathrm{cm}^{2}$ and a $\eta$ of $6.62 \%$ were measured. This $\mathrm{J}_{\mathrm{sc}}$ value is about $50 \%$ higher than that of the standard cell without any graphene. $49 \%$ gain in conversion efficiency was accomplished. The photovoltaic characteristics of the DSSCs with different graphene sizes are summarized in Table II. The performance enhancement of the graphene loaded cells was resulted mainly from the improvement in short circuit current.

The smaller graphene sheets could lead to larger surface area and higher porosity in the graphene- $\mathrm{TiO}_{2}$ composite films due to that their sizes are more compatible to the diameters of the $\mathrm{TiO}_{2}$ nanoparticles,

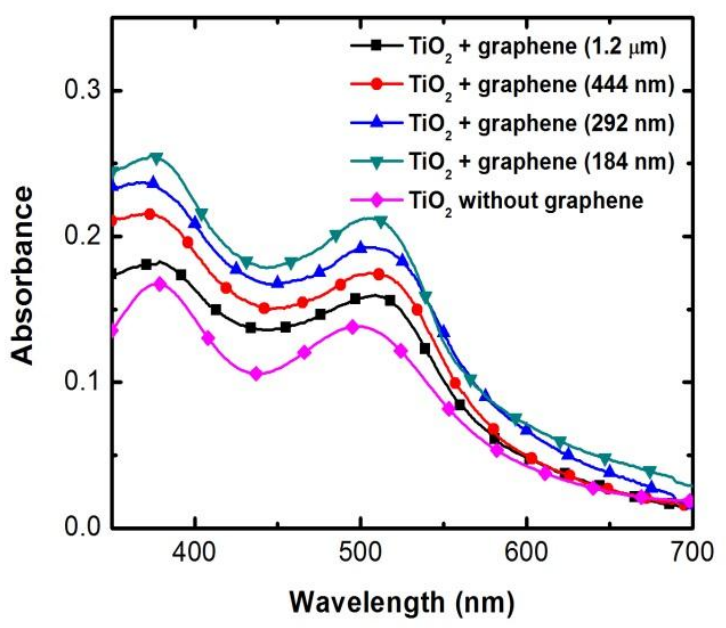

Figure 4. Absorption spectra of the solutions containing the amount of dye in aqueous $\mathrm{NaOH}$ removed from the photoanodes. 
resulting in higher dye adsorption. To confirm this, we measured the dye loadings in the composite films with the graphene sheets of various sizes along with a $\mathrm{TiO}_{2}$ film without any graphene by desorption of the dyes from the films in $0.1 \mathrm{M}$ aqueous $\mathrm{NaOH}$ solution. Fig. 4 shows the absorption spectra of the solutions, containing the amount of dyes in aqueous $\mathrm{NaOH}$ detached from the composite films and the $\mathrm{TiO}_{2}$ film. The area of all the films used was about $1 \mathrm{~cm}^{2}$. As the size of graphene sheet decreased, the dye loading also increased. It is known that the number of photogenerated electrons is directly related to the amount of dye loading in a DSSC. By decreasing the graphene sizes in the composite films, higher light harvesting efficiency was achieved, leading to larger $\mathrm{J}_{\mathrm{sc}}$. The smaller graphene sheets could be more compatible smaller graphene sheets to the $\mathrm{TiO}_{2}$ nanoparticles could have provided a more efficient pathway for the photogenerated electrons.

Table II. Photovoltaic properties of the DSSCs made with graphene of different sizes. (Double column)

\section{Conclusions}

In summary, we have investigated the photovoltaic performance of the DSSCs loaded with the graphene sheets of various sizes. It has been observed that as the sheet size decreases, the short circuit current increases. We speculate that the smaller graphene sheets could have helped in enhancement of the surface area and dye loading for better light-harvesting, leading to higher power conversion efficiency.

Acknowledgments. This work was supported as part of Polymer-Based Materials for Harvesting Solar Energy, an Energy Frontier Research Center funded by the U.S. Department of Energy, Office of Science, Basic Energy Sciences under Award \#DESC0001087.This research was also supported by in part by an appointment to the Faculty Research Participation Program at the U.S. Army Natick Soldier Research, Development and Engineering Center (NSRDEC) administered by the Oak Ridge Institute for Science and Education through and interagency agreement between the U.S. Department of Energy and
NSRDEC. Contributions from MJG \& SD were supported by the U.S. NSF under award CMMI1200489.

\section{References}

[1] B. O'regan, M. Grfitzeli, A low-cost, highefficiency solar cell based on dye-sensitized, Nature. 353 (1991) 24.

[2] A. Yella, H. Lee, H.N. Tsao, C. Yi, A.K. Chandiran, M.K. Nazeeruddin, E.W. Diau, C. Yeh, S.M. Zakeeruddin, M. Grätzel, Porphyrin-sensitized solar cells with cobalt (II/III)-based redox electrolyte exceed 12 percent efficiency, Science. 334 (2011) 629-634.

[3] S. Ito, S.M. Zakeeruddin, R. Humphry-Baker, P. Liska, R. Charvet, P. Comte, M.K. Nazeeruddin, P. Péchy, M. Takata, H. Miura, High-Efficiency OrganicDye-Sensitized Solar Cells Controlled by Nanocrystalline-TiO2 Electrode Thickness, Adv Mater. 18 (2006) 1202-1205.

[4] M. Yanagida, N. Onozawa-Komatsuzaki, M. Kurashige, K. Sayama, H. Sugihara, Optimization of tandem-structured dye-sensitized solar cell, Solar Energy Mater. Solar Cells. 94 (2010) 297-302.

[5] H.J. Snaith, L. Schmidt-Mende, Advances in Liquid-Electrolyte and Solid-State Dye-Sensitized Solar Cells, Adv Mater. 19 (2007) 3187-3200.

[6] Q. Zhang, G. Cao, Nanostructured photoelectrodes for dye-sensitized solar cells, Nano Today. 6 (2011) 91-109.

[7] C. Hagglund, M. Zach, B. Kasemo, Enhanced charge carrier generation in dye sensitized solar cells by nanoparticle plasmons, Appl. Phys. Lett. 92 (2008) 013113-013113-3.

[8] H.A. Atwater, A. Polman, Plasmonics for improved photovoltaic devices, Nature materials. 9 (2010) 205213.

[9] F. Schwierz, Graphene transistors, Nature nanotechnology. 5 (2010) 487-496.

[10] M.A. Worsley, S.O. Kucheyev, H.E. Mason, M.D. Merrill, B.P. Mayer, J. Lewicki, C.A. Valdez, M.E. Suss, M. Stadermann, P.J. Pauzauskie, Mechanically robust 3D graphene macroassembly with high surface area, Chem.Commun. 48 (2012) 8428-8430.

[11] H. Choi, H. Kim, S. Hwang, W. Choi, M. Jeon, Dye-sensitized solar cells using graphene-based carbon nano composite as counter electrode, Solar Energy Mater. Solar Cells. 95 (2011) 323-325.

[12] S. Sun, L. Gao, Y. Liu, Enhanced dye-sensitized solar cell using graphene-TiO photoanode prepared by heterogeneous coagulation, Appl. Phys. Lett. 96 (2010) 083113.

[13] H. Wang, S.L. Leonard, Y.H. Hu, Promoting Effect of Graphene on Dye-Sensitized Solar Cells, Ind 
Eng Chem Res. 51 (2012) 10613-10620.

[14] T. Tsai, S. Chiou, S. Chen, Enhancement of dyesensitized solar cells by using graphene-TiO2 composites as photoelectrochemical working electrode, Int.J.Electrochem.Sci. 6 (2011) 3333-3343.

[15] J. Song, Z. Yin, Z. Yang, P. Amaladass, S. Wu, J. Ye, Y. Zhao, W. Deng, H. Zhang, X. Liu, Enhancement of photogenerated electron transport in dye-sensitized solar cells with introduction of a reduced graphene oxide- $\mathrm{TiO} 2$ junction, Chemistry-A European Journal. 17 (2011) 10832-10837.

[16] J. Fan, S. Liu, J. Yu, Enhanced photovoltaic performance of dye-sensitized solar cells based on $\mathrm{TiO} 2$ nanosheets/graphene composite films, Journal of Materials Chemistry. 22 (2012) 17027-17036.
[17] W.S. Hummers Jr, R.E. Offeman, Preparation of graphitic oxide, J. Am. Chem. Soc. 80 (1958) 13391339.

[18] S. Das, F. Irin, H. Tanvir Ahmed, A.B. Cortinas, A.S. Wajid, D. Parviz, A.F. Jankowski, M. Kato, M.J. Green, Non-covalent functionalization of pristine fewlayer graphene using triphenylene derivatives for conductive poly (vinyl alcohol) composites, Polymer. 53 (2012) 2485-2494.

[19] M.S. Lee, I.C. Cheon, Y.I. Kim, Photoelectrochemical Studies of Nanocrystalline $\mathrm{TiO}_{2}$ Film Electrodes, BULLETIN-KOREAN CHEMICAL SOCIETY. 24 (2003) 1155-1162. 


\section{Graphical Abstract}

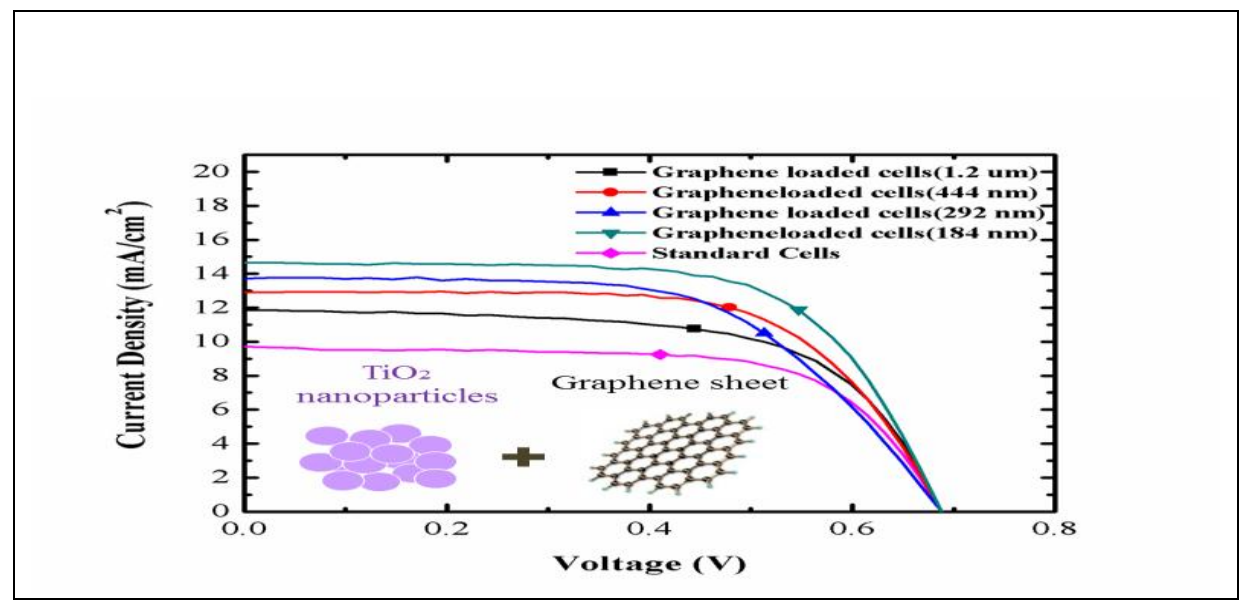

Table I. Sizes and monodispersities of the graphene sheets used in the DSSCs with or without sonication.

\begin{tabular}{|c|c|c|c|c|}
\hline Sonication time & $\begin{array}{c}\text { Hummers method }(0 \\
\text { min })\end{array}$ & Hummers method (30 min) & Hummers method (60 min) & Second method (0 min) \\
\hline Size & $1.2 \pm 0.11 \mathrm{um}$ & $444 \pm 55 \mathrm{~nm}$ & $292 \pm 20 \mathrm{~nm}$ & $184 \pm 15 \mathrm{~nm}$ \\
\hline
\end{tabular}

Table II. Photovoltaic properties of the DSSCs made with graphene of different sizes.

\begin{tabular}{|l|c|c|c|c|}
\hline DSSCs & $\mathrm{J}_{\mathrm{sc}}\left(\mathrm{mA} / \mathrm{cm}^{2}\right)$ & $\mathrm{V}_{\mathrm{oc}}(\mathrm{V})$ & FF & $\eta$ \\
\hline Standard cells & 9.76 & 0.68 & $66 \%$ & $4.43 \%$ \\
\hline Cells with Graphene $(1.2 \mu \mathrm{m})$ & 11.87 & 0.68 & $63 \%$ & $5.09 \%$ \\
\hline Cells with Graphene $(444 \mathrm{~nm})$ & 12.92 & 0.68 & $66 \%$ & $5.31 \%$ \\
\hline Cells with Graphene $(292 \mathrm{~nm})$ & 13.75 & 0.68 & $60 \%$ & $6.15 \%$ \\
\hline Cells with Graphene $(184 \mathrm{~nm})$ & 14.66 & 0.68 & $66 \%$ & $6.62 \%$ \\
\hline
\end{tabular}

